\title{
Tagungsankündigungen
}

\section{1th International Symposium on Microchemical Techniques}

Zeit: $28 .-08$. bis 01.09 .1989

Ort: Wiesbaden

Vorsitzender: Wilhelm FRESENIUS, Taunusstein

Information:

Das Programm ist ab Mai 1989 über die GDCh-Geschäftsstelle/Abteilung Tagungen erhältlich. Telefon: 069/79 17-3 66

\section{Programmübersicht}

Festvortrag: Order and Chaos in Chemistry and Biology

B. Hess, Dortmund

Plenarvorträge: Inorganic Mass Spectrometry of Solid Samples

F. ADAMS, Wilrijk, B

High Performance Mass Spectrometry of Sputtered Material: A Powerful Tool for Inorganic and Organic Trace, Micro and Surface Analysis

A. Benninghoven, Münster

Surface and Thin Film Analysis - Comparison of Methods and Trends

H. W. WERNER, Eindhoven, NL

Critical Comparison of Plasma Excitation, Sources (ICP, MIP, CMP, DCP a.o.) in Optical Emission Spectroscopy

H. M. HIEFTJE, Bloomington, USA

Instrumental Developments in Organic Mass Spectrometry

M. LINSCHEID, Dortmund

Prechromatographical Micro Methods in Organic Trace Analysis - Critical Survey

R. W. FreI, Amsterdam, NL

Chemometrics - its Role in the Analytical Laboratory

B. G. M. VANDEGINSTE, Nijmengen, NL
Analytical Methods in Environmental Chemistry

O. Hutzinger, Bayreuth

Hauptvorträge:

Methoden der Elementanalyse

Optische Spektroskopie

Elektronenmikroskopie

Röntgenspektrometrie

Massenspektrometrie der Elemente

Elektrochemische Analyse

Flow-Injection Analysis

Ionenchromatographie

Kernchemische Methoden

Probenvorbereitung in der Spuren-

analyse

Speciation Analysis

Methoden der Organischen Analyse

Chromatographie

Molekülspektroskopie

Organische Massenspektrometrie

Organische Mikroelementaranalyse

Sensoren

Chemometrics

Roboter

Angewandte Mikro- und Spurenanalyse

Materialwissenschaften

Agrikulturchemie

Lebensmittelchemie

Umweltanalytik

Biotechnologie

Gerichtschemie

Archäometrie

Podiumsdiskussion: Reproduzierbarkeit der Analysenergebnisse Ausstellung: stellung, bei der Geräte für die diskutierten Techniken und neue Literatur der tangierten Fachgebiete präsentiert werden.

$\begin{array}{ll}\text { Analysis and Evaluation of Atmospheric } \mathrm{CO}_{2} \text { Data: Present and Past } \\ \text { - Third International } \mathrm{CO}_{2} \text { Conference } & \\ \text { Time: October } 16-23,1989 & \text { Phone: 49-6221-563330, Telefax: 49-6221-563111 } \\ \text { Place: Hinterzarten (Black Forest) FRG } & \text { or preferably } 563405 \text {; Telex: } 461745 \text { uni kl } \\ \text { For more information: } & \text { Scientific Program: } \\ \text { Dr. Ingeborg Levin, } & \text { 1. Techniques for } \mathrm{CO}_{2} \text { data assessment } \\ \text { Institut für Umweltphysik } & \text { 2. Interpretation of temporal and spatial variations of atmospheric } \\ \text { Universität Heidelberg } & \mathrm{CO}_{2} \text { concentration and isotopic composition } \\ \text { Im Neuheimer Feld } 366 & \text { 3. Modelling of the carbon cycle } \\ \text { D-6900 Heidelberg, FRG } & \text { 4. Long-term changes in the carbon cycle }\end{array}$

\section{Chemische Gesellschaft der DDR}

\section{- Hauptjahrestagung}

Zeit: 29. 11. - 01. 12. 1989

Ort: Karl-Marx-Stadt

Themenbereich:

Die Tagung ist vor allem Problemen der Festkörper- und Grenzflächenchemie gewidmet sowie aktuellen Fragen:
- Chemie der Werkstoffentwicklung und -veredelung,

- Chemie der Rohstoffbereitstellung,

- Hochveredelungschemie,

- Recyclingprozesse,

- Abproduktverwertung und chemische Probleme des Umweltschutzes,

- Einsätze von Computern in der Chemie. 